Meta

Journal des traducteurs

Translators' Journal

\title{
Prolégomènes à la typologie de textes en interprétation simultanée
}

\section{Henri Niedzielski}

Volume 33, numéro 4, décembre 1988

Symposium AILA 1987, Sydney

URI : https://id.erudit.org/iderudit/003734ar

DOI : https://doi.org/10.7202/003734ar

Aller au sommaire du numéro

Éditeur(s)

Les Presses de l'Université de Montréal

ISSN

0026-0452 (imprimé)

1492-1421 (numérique)

Découvrir la revue

Citer cet article

Niedzielski, H. (1988). Prolégomènes à la typologie de textes en interprétation simultanée. Meta, 33(4), 491-496. https://doi.org/10.7202/003734ar d'utilisation que vous pouvez consulter en ligne.

https://apropos.erudit.org/fr/usagers/politique-dutilisation/ 


\section{PROLÉGOMĖNES À LA TYPOLOGIE DE TEXTES EN INTERPRÉTATION SIMULTANÉE 1}

HENRI NIEDZIELSKI

Université d'Hawaii, Honolulu, USA

\section{DISCOURS DIRIGÉ DE L'INTERPRÈTE}

Dans une autre communication (Niedzielski 1987), j'ai introduit la notion de discours dirigé de l'interprète, une sorte d'hybride entre le discours interactionnel de la conversation et le discours autonome d'un texte rédigé. Dans sa forme, le discours dirigé est oral et généralement moins libre que celui de la conférence magistrale. Dans son déroulement sémantique, il est totalement assujetti à l'évolution de la pensée de l'orateur. En effet,

dans le jeu de la parole traduisante, le traducteur doit construire un espace mental, résultat d'une saisie du sens intenté par l'orateur/auteur pendant un premier acte de parole réalisé dans la langue de l'orateur/auteur et le communiquer à un tiers dans un deuxième acte de parole réalisé dans la langue du tiers (Garcia-Landa $1985: 36$ ).

L'interprète doit donc être à la fois un excellent auditeur et un locuteur accompli pour pouvoir rester fidèle non seulement à la pensée de l'orateur mais aussi au style de son discours. D'après Déjean Le Féal (1985:55), " l'inobservation du registre original conduit à une infidélité de la traduction plus grave et plus lourde de conséquences que celle causée par une erreur matérielle que l'interprète pourrait commettre ". À la variété des registres - texte littéraire, texte philosophique, texte publicitaire - vient s'ajouter la diversité des modes de présentation de l'orateur. Certaines caractéristiques de texte suggèrent qu'il est possible de dresser une typologie de textes en interprétation simultanée selon les difficultés présentées par le fond ou la forme.

\section{HUIT DIMENSIONS DE TEXTUALITÉ}

La typologie proposée ci-dessous s'inspire des caractéristiques de textes déjà décrites par Kopczyński et relevées par Bühler (1986). Le but de cette typologie est d'aider les apprentis interprètes et, par conséquent, elle est accompagnée d'exercices pratiques qui visent à réduire les difficultés causées par huit dimensions de textualité 2 .

Afin de mieux comprendre comment ces huit dimensions de textualité influencent la tâche de l'interprète, il est nécessaire d'en donner une brève définition.

La cohésion est plus ou moins synonyme de l'organisation logique d'un discours : le degré de cohésion d'un discours est d'autant plus élevé que son articulation apparaît plus clairement dans la progression de ses unités reliées par des formules de transition très nettes.

La cohérence se définit par la facilité avec laquelle des structures morphosyntaxiques ou phonologiques évoquent le contenu sémantique logique d'un discours. 
L'intentionalité concerne le but recherché par l'auteur du discours : information, recrutement ou appréciation esthétique. Ces trois buts différents servent souvent de base à une typologie de textes (cf. Newmark $1981: 13-15$ ).

La situationalité détermine si le contenu d'un discours, ou éventuellement sa forme, est bien choisi en fonction du public auquel l'orateur s'adresse.

La densité d'information est le produit mathématique de la longueur du discours, de sa densité sémantique, de sa complexité morphosyntaxique, de sa clarté acoustique et de sa vitesse de présentation.

L'informativité se mesure par la quantité de concepts nouveaux présentés au public - une surabondance d'éléments inconnus ou nouveaux risque de décourager alors qu'une disette entraîne un manque d'intérêt.

L'acceptabilité d'un discours traduit l'attitude du public. Celui-ci peut accepter ou rejeter le discours au niveau de la pensée — pour des raisons sociolinguistiques - ou à celui de la structure - pour des raisons linguistiques. Cependant, en interprétation simultanée le public fait en général preuve de très grande tolérance linguistique.

L'intertextualité est l'ensemble des facteurs qui permettent de comprendre un discours grâce à la connaissance antérieure d'autres textes. "Les sens des discours sont ainsi perçus grâce à la jonction d'actualisations sémiques à des savoirs antérieurs » (Seleskovitch $1985: 22$ ).

Dans le contexte de l'interprétation simultanée, le lieu de ces huit dimensions semble se concentrer comme suit : la cohésion et la cohérence explicitent le contenu linguistique du message ; l'intentionalité, la situationalité et la densité d'information sont choisies par le conférencier ; l'informativité et l'acceptabilité varient avec le public alors que l'intertextualité dépend beaucoup de l'interprète.

\section{PARADOXE DU DISCOURS DIRIGÉ}

Bien qu'idéalement, l'interprète devrait transférer fidèlement tous les sens d'un message tout en en conservant le style, il doit parfois améliorer une ou plusieurs des dimensions de textualité. En effet, son public n'entend que lui et n'a aucun moyen de savoir que c'est le conférencier lui-même qui "s'exprime mal " (Niedzielski 1985 : 277). Ce labeur d'amélioration est d'autant plus nécessaire que l'orateur parle plus spontanément. Comme, d'autre part, l'interprétation simultanée est d'autant plus difficile que le style du texte à traduire est plus recherché, la forme du discours dirigé de l'interprète tend à se rapprocher de celle du discours de la conversation quand l'orateur lit son texte et de celle du discours rédigé quand il le parle. Le paradoxe de ces deux tendances opposées qui confèrent au discours dirigé son apparence d'intermédiaire détermine la première distinction dans notre typologie entre les textes oraux et les textes écrits.

\section{TEXTES ORAUX}

Une grande diversité existe entre les textes oraux selon leur domaine, leur registre, leur longueur et le nombre de locuteurs.

\subsection{LE MONOLOGUE IMPROMPTU}

Les textes oraux les plus faciles à traduire sont souvent les plus courts, telle une allocution impromptue de bienvenue ou la présentation d'un conférencier.

Des difficultés peuvent néanmoins se présenter à l'interprète parce que le texte de l'annonciateur peut lui paraître manquer de cohérence à cause de certains noms peu familiers ou ne pas être acceptable tel quel à cause de normes culturelles différentes dans les deux langues. 
Les exercices recommandés ${ }^{3}$ pour surmonter les obstacles imposés par le manque apparent de cohérence comprennent la notation phonétique en plusieurs étapes voyelles, consonnes, syllabification - (cf. Niedzielski 1975). Quant à l'acceptabilité, elle peut s'améliorer par la paraphrase ou la transposition.

\subsection{LE MONOLOGUE PRÉPARÉ NON RÉDIGÉ}

S'appuyant souvent sur des notes, ce genre de monologue s'entend, entre autres, au tribunal lorsque le juge prononce sa sentence, ou dans une assemblée lorsque le président de séance résume une résolution.

L'informativité prime sur toutes les autres dimensions d'un tel texte. Le transfert précis de tous les éléments d'information du message exige une excellente précompréhension du sujet et de la situation. D'où l'importance de l'intertextualité.

L'acquisition de terminologies spécifiques est indispensable. Elle peut se réaliser directement par l'apprentissage d'associations syntagmatiques dites "collocations" dans les deux langues et indirectement par la pratique des associations lexicales libres. En fait, "des opérations traduisantes répétées créent des associations qui s'engramment dans la mémoire" (Pergnier $1980: 474)$.

\subsection{LE DIALOGUE PARTIELLEMENT PRÉPARÉ}

Certaines sociétés utilisent l'interprétation par téléphone. Par exemple, un dialogue se déroule sur la voie publique en Californie entre un agent de police ou un ambulancier d'une part, et un détenu ou un blessé d'autre part. L'interprète se trouve à Hawaï et l'interprétation est consécutive mais il arrive qu'elle soit instantanée.

Aux difficultés déjà nommées ci-dessus vient s'ajouter une densité d'information élevée à cause du bruit parasitique au téléphone.

En plus des activités déjà décrites, l'étudiant peut faire des exercices d'écoute programmés à clarté et précision décroissantes (Niedzielski 1983).

\subsection{LE MONOLOGUE PRÉPARÉ ET PARTIELLEMENT RÉDIGÉ}

Dans ce genre de monologue, le débit a tendance à être irrégulier : rapide là où le conférencier lit ou récite par coeur, hésitant et lent aux transitions articulatoires. Cette situation est assez courante dans les milieux académiques. Elle se produit aussi dans les conférences internationales politiques ou autres lors de la présentation du programme général de la rencontre ou des activités de la journée.

Une densité d'information énorme causée par une rapidité du débit excessive par intermittence ainsi qu'une cohésion déficiente à cause d'une articulation globale du monologue difficile à reconnaître représentent des problèmes spécifiques de ce genre de monologue.

L'effort accru exigé de l'interprète pendant les épisodes particulièrement denses peut être compensé en partie par un allègement de son fardeau d'explicitation de l'articulation. Des exercices de reconnaissance puis de création d'un plan aident à définir les macrostructures d'un texte. Pour rendre leur enchaînement plus clair, il est utile d'apprendre systématiquement les expressions formules typiques pour les sujets traités et les formules de transition logique dans les deux langues.

\subsection{LE MONOLOGUE APPRIS ET RÉCITÉ}

Plusieurs formes d'un tel monologue existent : conférence d'information d'un professionnel très spécialisé, allocution de motivation d'un animateur pour encourager des vendeurs, récitation d'un conte à morale pour illustrer une thèse, discours politique.

Bien que l'interprète ne doive jamais exprimer les intentions des orateurs, il est obligé de les reconnaître afin de pouvoir les transférer en $\mathrm{L}_{2}$. En effet, "les intentions des orateurs, l'objectif poursuivi par leurs interventions - vouloir plaire ou, au contraire, 
menacer, flatter, concéder, faire rire, convaincre - sous-tendent leurs dires sans être verbalisées " (Seleskovitch 1985 : 23). Cette nécessité d'analyser l'intentionalité du discours s'applique évidemment à tous les genres de textes, mais la tâche est sans doute la plus ardue pour ce genre de monologue car il est souvent vocatif ou esthétique.

Puisque " l'enfer est pavé de bonnes intentions ", il arrive que l'orateur choisisse un texte inapproprié à la situation ou inacceptable au public. Une des supériorités de l'interprète sur l'ordinateur c'est précisément sa faculté d'évaluer le public et, par suite, d'apprécier la situationalité et l'acceptabilité du texte de l'orateur. Doit-il ou non, par exemple, traduire une histoire raciste ? Sans doute pas si un de ses rôles est celui d'ambassadeur ou de faciliteur d'échanges. "Sur ce droit de trahir pour servir reposent beaucoup de polémiques autour de la traduction" (Pergnier 1980:331).

Cette évaluation par l'interprète de l'intentionalité, de la situationalité et de l'acceptabilité du texte (ou de certaines de ses composantes) doit se faire pour ainsi dire instantanément car la rapidité du débit et la richesse de la prosodie alourdissent la densité d'information et ne laissent guère de temps à l'interprète pour réfléchir.

Grâce à des analyses de texte variées, l'interprète peut apprendre à découvrir très vite les intentions d'un orateur, y compris celles d'un propagandiste. Des études sociolinguistiques des deux communautés linguistiques peuvent le préparer à évaluer les situations et à décider ce qui est acceptable ou non.

\subsection{LE TEXTE ENREGISTRÉ}

Les données techniques sont souvent accompagnées de diapositives ou de films. Ces supports visuels sont parfois synchronisés avec un texte enregistré.

Le souci primordial de ces présentations est l'information. Cependant, même si l'interprète voit les projections - ce qui n'est malheureusement pas toujours le cas - il lui sera parfois difficile d'interpréter certains graphiques ou diagrammes.

La solution idéale se produit très rarement. Une seule fois ai-je eu la chance d'être convoqué la veille de la séance de travail pour synchroniser avec un long film publicitaire ma lecture du commentaire déjà traduit pour moi. De plus, toutes les expressions clés étaient déjà soulignées.

Faute de cet idéal, deux sortes d'exercices peuvent s'avérer très utiles : la description de représentations visuelles et la mémorisation de séries de chiffres. L'interprétation de supports visuels demande aussi l'apprentissage d'expressions toutes faites tirées de la terminologie appropriée. Dans les exercices de mémorisation avec chiffres, il s'agit de découvrir des moyens mnémotechniques efficaces pour un individu donné. Par exemple, dans une série de sept chiffres ou plus, certaines personnes se souviennent mieux des premiers, d'autres personnes des derniers, mais toutes éprouvent plus de difficulté à se rappeler les chiffres isolés (ex : 9, 4,6,5,8,7,0 plutôt que 946, 58, 70). Les séries peuvent être constituées d'autres éléments que des chiffres.

\section{TEXTES ÉCRITS}

La plupart des communications présentées dans les réunions internationales se situent à un niveau de langue moyen parce que les orateurs et le public possèdent une éducation supérieure, sans être des grammairiens, des stylistes ou des poètes. Certaines exceptions se produisent lorsque, par exemple, l'orateur se sert d'un texte entièrement rédigé. Dès lors, sa langue est plus soutenue et parsemée des complexités caractéristiques de la langue écrite.

\subsection{LE MONOLOGUE RÉDIGÉ ET RÉCITÉ}

Caractéristique sans doute de la majorité des communications académiques, ce genre de monologue peut se rencontrer aussi sous différentes formes dans le monde des 
affaires (présentation, prière, allocution, poème). Par exemple, le président directeur général d'une société de vente à domicile du Canada aime commencer les réunions générales annuelles par une prière qu'il lit comme s'il la récitait. La directrice du service des ventes de la même société composa un poème pour encourager les représentants à mieux vendre.

Un tel monologue peut contenir des noms propres et des expressions idiomatiques ou stylistiques qui entravent la cohérence et augmentent la densité d'information. En outre, des raisons sociolinguistiques peuvent exiger des adaptations (une autre prière, d'autres images) pour améliorer la cohérence du texte et son acceptabilité.

En plus des techniques déjà mentionnées, l'interprète se trouve parfois forcé d'utiliser le résumé. Il peut en maîtriser l'art en s'exerçant d'abord dans sa meilleure langue, puis dans l'autre, et enfin en résumant directement en $L_{2}$ des textes écrits en $L_{1}$.

\subsection{TEXTE RÉGIGÉ ET LU}

Sans compter les communications lues alors qu'elles devraient être racontées, ce genre de texte présente au moins deux variantes : la citation et le document officiel.

Si l'orateur ne ralentit pas son débit de lecture, l'interprète peut éprouver des difficultés assez considérables, parfois même s'il a pu traduire le texte à l'avance.

La traduction orale en $L_{2}$, courante et de plus en plus rapide, de textes écrits en $L_{1}$ et de plus en plus difficiles peut préparer l'interprète aux situations où il reçoit le texte en $\mathrm{L}_{1}$ à la dernière minute, c'est-à-dire au moment où il doit le traduire en $\mathrm{L}_{2}$. Dans les cas où il ne reçoit aucun texte, sa seule planche de salut est parfois le résumé. D'une façon générale, il est possible d'augmenter sa vitesse d'interprétation en s'exerçant à la lecture rapide et à la lecture d'un texte en $\mathrm{L}_{2}$ synchronisée avec l'écoute du texte correspondant en $L_{1}$.

\section{CONCLUSION}

La typologie proposée ci-dessus pour les textes en interprétation simultanée n'est sans doute pas complète mais elle s'appuie sur une expérience pratique de plusieurs décennies et pourrait constituer la première étape d'une expérimentation scientifique.

Il existe des interprètes privilégiés qui habitent au bon endroit et peuvent se spécialiser. Il en est d'autres qui non seulement n'ont guère de choix en la matière mais qui souvent doivent travailler seuls. Pour affronter la vaste diversité des tâches qui les attend, ces interprètes en simultanée généralistes ont besoin, encore plus que les autres, de certaines aptitudes ou connaissances fondamentales - bilinguisme absolu, bonne audition, élocution claire et agréable, mémoire à court terme supérieure, talent d'acteur, grande résistance physique et nerveuse, vaste savoir encyclopédique, facilité d'apprentissage et d'adaptation (Niedzielski 1985).

Étant donné que les aptitudes varient d'un individu à un autre et que les difficultés d'interprétation dépendent des deux langues de travail, le programme d'études en interprétation simultanée doit être complètement individualisé. Néanmoins, le curriculum pourrait se fonder sur les exercices décrits ci-dessus. De plus, avant même de soumettre un étudiant à ces études ardues, il serait bon d'évaluer ses aptitudes en lui faisant essayer ces exercices pendant un semestre de propédeutique.

Il est même concevable qu'un test d'aptitude à l'interprétation (simultanée) pourrait être élaboré à partir de ces exercices issus de la typologie proposée.

\section{Notes}

1. Le présent article est une version augmentée d'une intervention présentée au congrès de l'AILA en août 1987. 
2. J'ajoute " densité d’information " aux sept dimensions - cohésion, cohérence, intentionalité, acceptabilité, informativité, situationalité et intertextualité - déjà analysées par Beaugrande et Dressler dans leur Introduction to Text Linguistics, 1981, London \& New York, Longman.

3. Les exercices recommandés pour un genre de texte sont choisis parce qu'ils aident à surmonter des obstacles spécifiques pour ce genre de texte. Ils n'excluent pas d'autres exercices décrits à l'occasion d'un autre genre de texte présentant des caractéristiques ou des difficultés semblables. La liste de tous les exercices n'est pas non plus complète et le lecteur peut y en ajouter d'autres.

\section{RÉFÉRENCES}

BEAUGRANDE, R.-A. de \& W.U. DRESSLER (1981) : Introduction to Text Linguistics, London \& New York, Longman

BÜHLER, H. (1986) : "Text Linguistics and the Spoken Text ", version dactylographiée.

DÉJEAN LE FÉAL, K. (1985) : "Le registre littéraire en interprétation simultanée ", META, 30:1, pp. 55-64.

GARCIA-LANDA, M. (1985) : "L'oralité de la traduction orale », META, 30:1, pp. 30-36.

NEWMARK, P. (1981) : Approaches to Translation, Oxford, Pergamon Press.

NIEDZIELSKI, H. (1975) : "Apprentissage de la compréhension orale au laboratoire de langues ", Leeds, Eighth International Congress of Phonetic Sciences, communication orale.

NIEDZIELSKI, H. (1983) : "Teaching Natural Speech Understanding ", Studia Anglica Posnaniensia, xvi, pp. 205-231.

NIEDZIELSKI, H. (1985) : "Teaching Simultaneous Interpreting to Bilingual Students of Business English ", Kwartalnik Neofilologiczny, xxx, 3, pp. 277-286.

NIEDZIELSKI, H. (1987) : "Formulaic Speech in the Teaching of Controlled Discourse to Apprentice Interpreters ", Hildesheim, AILA Symposium on Text Linguistics and Languages for Special Purposes, à paraître.

PERGNIER, M. (1980) : les Fondements sociolinguistiques de la traduction, Paris, Champion.

SELESKOVITCH, D. (1985) : "Interprétation ou interprétariat ? ", META, 30:1, pp. 19-24. 